\title{
Tolerance has its limits: how the thymus copes with infection
}

\author{
Cláudio Nunes-Alves ${ }^{1,2,3}$, Claudia Nobrega ${ }^{1,2}$, Samuel M. Behar ${ }^{3}$, and \\ Margarida Correia-Neves ${ }^{1,2}$
}

\author{
${ }^{1}$ Life and Health Sciences Research Institute (ICVS), School of Health Sciences, University of Minho, $4710-057$ Braga, Portugal \\ ${ }^{2}$ ICVS/3B's - PT Government Associate Laboratory, Braga/Guimarães, Portugal \\ ${ }^{3}$ Department of Microbiology and Physiological Systems, University of Massachusetts Medical School, Worcester, MA, USA
}

\begin{abstract}
The thymus is required for $\mathrm{T}$ cell differentiation; a process that depends on which antigens are encountered by thymocytes, the environment surrounding the differentiating cells, and the thymic architecture. These features are altered by local infection of the thymus and by the inflammatory mediators that accompany systemic infection. Although once believed to be an immune privileged site, it is now known that antimicrobial responses are recruited to the thymus. Resolving infection in the thymus is important because chronic persistence of microbes impairs the differentiation of pathogen-specific $T$ cells and diminishes resistance to infection. Understanding how these mechanisms contribute to disease susceptibility, particularly in infants with developing $T$ cell repertoires, requires further investigation.
\end{abstract}

\section{The thymus is essential for $\mathbf{T}$ cell differentiation}

The appearance of adaptive immunity in jawed vertebrates is considered a major evolutionary step, because B and T cells enable the immune system to generate and recall pathogen-specific immune responses [1]. Among lymphocytes, $\mathrm{T}$ cells are unique in their expression of the $\mathrm{T}$ cell receptor (TCR). TCR-mediated recognition of microbial peptides bound to major histocompatibility complex (MHC) is the principle way that the immune system identifies infected cells. T cell precursors are generated in the bone marrow and become functional after differentiation within the thymus (Figure 1) [1]. The deceptively simple anatomical structure of the thymus belies its sophisticated ability to generate $\mathrm{T}$ cells expressing a broad TCR repertoire capable of recognizing virtually any foreign antigen. Thymic selection eliminates most potentially harmful selfreactive $\mathrm{T}$ cells. After selection, naïve self-restricted $\mathrm{T}$ cells exit the thymus and traffic to secondary lymphoid organs.

$\mathrm{T}$ cell differentiation depends on the thymic microenvironment and the cytokine milieu surrounding the differentiating cells [1]. This raises the possibility that during infection, changes in soluble factors or antigens present within the thymus alter $\mathrm{T}$ cell differentiation. Indeed, systemic infection has detrimental effects on thymic structure

Corresponding authors: Behar, S.M. (samuel.behar@umassmed.edu);

Correia-Neves, M. (mcorreianeves@ecsaude.uminho.pt).

Keywords: thymus; infection; T cell repertoire; antimicrobial response;

thymic microenvironment.

$1471-4906 / \$$ - see front matter

(c) 2013 Elsevier Ltd. All rights reserved. http://dx.doi.org/10.1016/j.it.2013.06.004 and function (Figure 2). Furthermore, certain bacteria, virus, fungi, and parasites can directly invade the thymus (Table 1). These observations suggest that some pathogens, particularly those that cause chronic infections, interfere with the generation of immune responses designed to fight them by disrupting $\mathrm{T}$ cell development and possibly altering central tolerance. Here, we discuss how attitudes about thymic function during infection are changing. It is now clear that diverse pathogens infect the thymus, and because of their local and systemic effects, disrupt its architecture and function. We review recent evidence that the presence of pathogens in the thymus leads to microbe-specific tolerance and impairs host resistance. However, the immune system reacts to such invasions by recruiting immune responses to the thymus. Finally, we suggest that the ability to defend the thymus from infection may be important in maintaining the integrity of the immune system, particularly in situations where the $\mathrm{T}$ cell repertoire is being generated or is regenerating, such as in young children or patients with AIDS, respectively.

\section{Thymus: myth and reality}

Few studies have addressed whether infection affects thymic function in people, with the exception of studies on HIV infection [2]. In part, this is because of limited availability of thymus from infected patients, because the thymus is difficult to biopsy, and the usefulness of indirect measurements of thymic activity in humans is still controversial. As important are misconceptions that have affected how scientists and physicians view thymic function. Although now largely refuted, these include: (i) the thymus is an immune-privileged site protected from infection and immune responses; and (ii) thymic function is only important during early life and dispensable after puberty.

The notion that the thymus is immune privileged is inseparable from the concept of the blood-thymus barrier; considered for several years to be responsible for an antigen-free thymic microenvironment that prevents immune responses to exogenous antigens [3]. However, it is now clear that the thymus is both a target of infection and a site to where immune responses are recruited (Table 1) [4-7].

Although the thymus is essential for the establishment of a diverse $\mathrm{T}$ cell repertoire early in life, it is widely believed to be unnecessary after puberty. This idea is supported by the finding that the peripheral $\mathrm{T}$ cell pool can be maintained by thymic-independent mechanisms [8]. Even if this were 


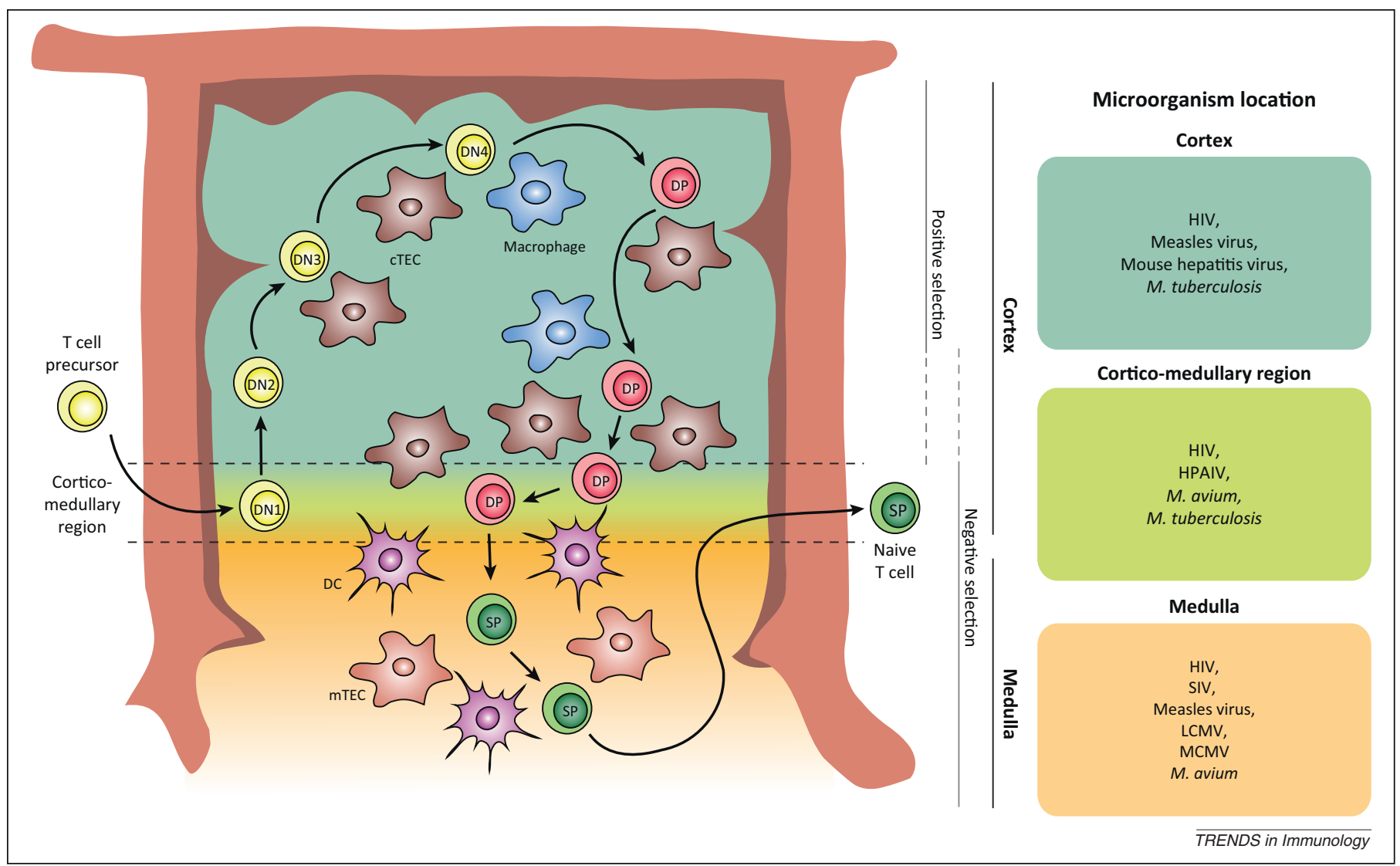

Figure 1. T cell differentiation. Schematic representation of T cell traffic within the thymus and location of the major steps during $T$ cell selection. T cell precursors from the bone marrow enter the thymus in the cortico-medullary region and migrate to the cortex, where they go through the double negative (DN; e.g., $\mathrm{CD}^{-} \mathrm{CD}^{-}{ }^{-}$) stages of $\mathrm{T}$ cell differentiation and become double positive (DP; e.g., $C D 4^{+} \mathrm{CD} 8^{+}$) cells. DP cells migrate from the cortex to the medulla, interacting with structural components of the thymus

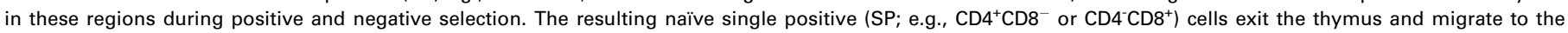
peripheral lymphoid organs. Several microbes can be detected within the thymus following infection. Examples of microbes that are detected in the cortex, medulla or cortico-medullary region of the thymus following in vivo infection are shown. Abbreviations: DC, dendritic cell; DN, double negative thymocytes; DP, double positive thymocytes; SP, single positive thymocytes; cTEC, cortical thymic epithelial cell; mTEC, medullary thymic epithelial cell.

true, it is incredibly shortsighted to ignore the effect of infection on the thymus, because children and young adults under the age of 24 years make up $44 \%$ of the world's population, and are particularly vulnerable to infection. In 2011, nearly 6.9 million children under the age of 5 years died, with infection causing more than half of these deaths [9]. Although most of these deaths were due to acute infection (pneumonia, $14 \%$; diarrhea, $10 \%$; measles, $1 \%$ ), a significant number were due to chronic infection (malaria, 7\%; HIV/AIDS, 2\%; tuberculosis, 1\%) [9]. These numbers increase with age and HIV/AIDS and tuberculosis account for $11 \%$ of deaths among young adults (age 10-24 years) worldwide [9]. Both HIV and Mycobacterium tuberculosis infect the thymus and cause alterations in $\mathrm{T}$ cell output, which could be relevant both in settings of vaccination and during natural immunity to these pathogens [2,10-12].

Although childhood infections are arguably a greater cause of morbidity and mortality than adult infection, particularly in the developing world, recent studies reinforce the idea that the thymus affects resistance to infection during adulthood. Reduced thymic output of $\mathrm{T}$ cells is associated with HIV progression to AIDS and the thymus has been implicated in the successful immune reconstitution of AIDS patients in response to antiretroviral therapy [2]. Moreover, thymectomy during early childhood has been linked to accelerated decline in immunologic function, particularly following cytomegalovirus (CMV) infection [13], and work on experimental viral infection models finds that continuous recruitment of naïve $\mathrm{T}$ cells from the thymus has a beneficial role in the control of persistent infections [14-16]. The integrity of the adult thymus is also required for other aspects of ongoing immunity including antibody generation [17] and oral tolerance [18]. These observations indicate that an intact and functional thymus is required for optimal immunity to infection throughout life.

\section{How does infection alter thymic function?}

There are two ways in which infection can affect the thymus: local and systemic (Figure 2). Local refers to effects of direct infection of the thymus by microbes. Systemic refers to the consequences of infection elsewhere on the thymus. Systemic effects occur when soluble factors, such as glucocorticoids (GCs) and proinflammatory mediators, are released into the blood stream.

\section{Infection-induced thymic atrophy}

Premature thymic atrophy is a common consequence of infection by viruses, bacteria, parasites, and fungi (Box 1 and Figure 2) [19] and can result from local and/or systemic effects. For example, GC levels rise during acute infection, and can induce thymocyte apoptosis, especially among 


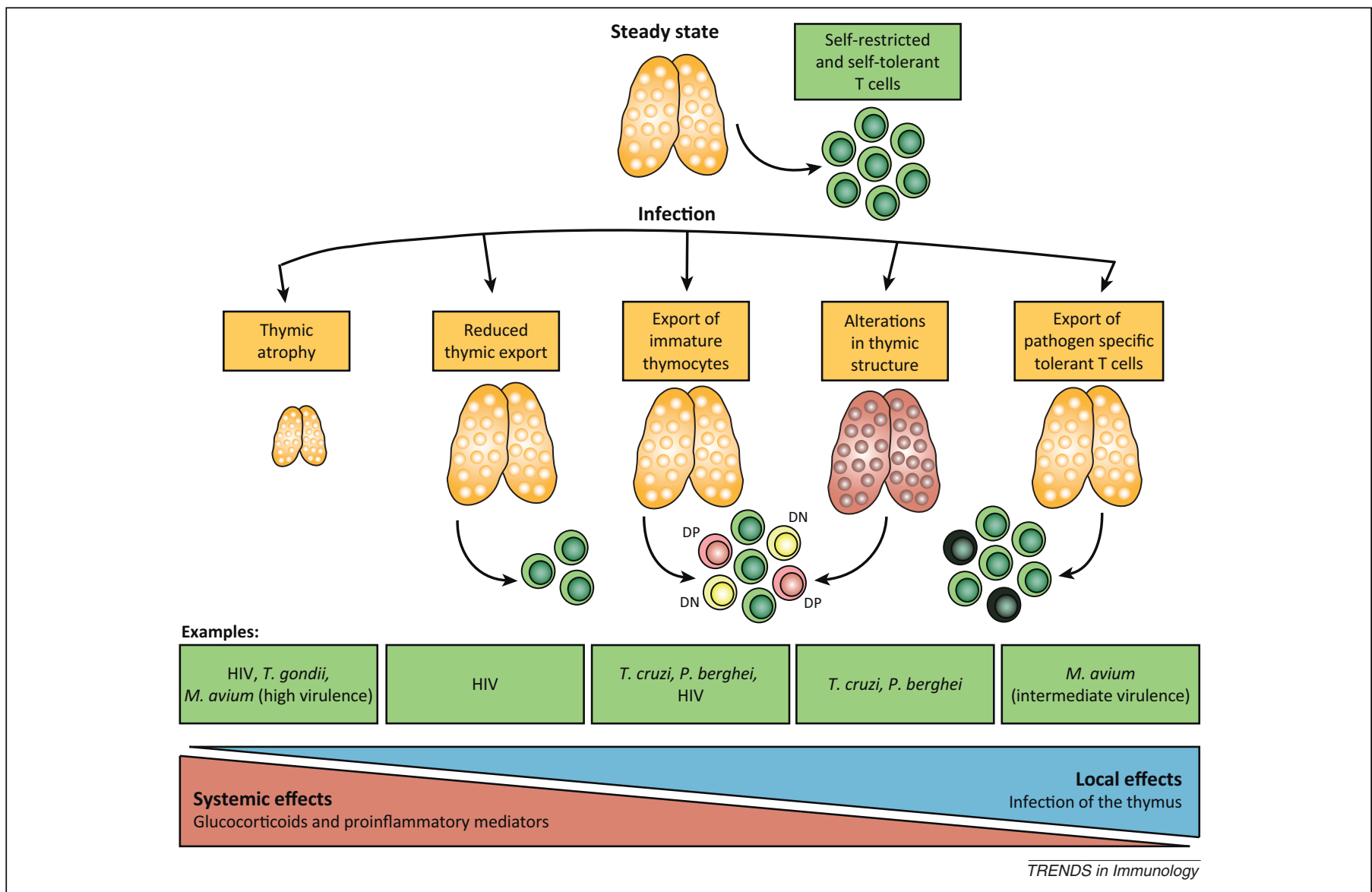

Figure 2. The effects of infection on the thymus. Schematic representation of how infection can affect the thymus through systemic and/or local effects. Glucocorticoids and/or proinflammatory mediators mediate systemic effects, while local effects require the presence of a pathogen within the thymus. Infection-induced alterations include thymic atrophy, modifications in the thymic structure, and alterations in the T cells exported to the periphery. Representative pathogens capable of inducing the different alterations in thymic structure and/or function are indicated. Abbreviations: DN, double negative thymocytes; DP, double positive thymocytes.

Table 1. Pathogens that infect the thymus ${ }^{a}$.

\begin{tabular}{|c|c|c|c|}
\hline & Pathogen & Consequences of infection & Refs \\
\hline \multirow{8}{*}{ Virus } & HIV & Atrophy & {$[2,36-40]$} \\
\hline & Influenza virus & Atrophy (strain dependent); immune response in the thymus & [7] \\
\hline & Murine leukemia virus (MLV) & Atrophy; pathogen-specific immune tolerance & {$[42,43,48]$} \\
\hline & Mouse hepatitis virus & Atrophy & [50] \\
\hline & Coxsackievirus & Modulation of TEC function & [52] \\
\hline & Epstein-Barr virus (EBV) & N/A & [71] \\
\hline & Junin virus & $\mathrm{N} / \mathrm{A}$ & [72] \\
\hline & Poliovirus & N/A & [73] \\
\hline Bacteria & Mycobacterium avium & $\begin{array}{l}\text { Atrophy (strain dependent); immune response in the thymus; } \\
\text { pathogen-specific immune tolerance }\end{array}$ & $\begin{array}{l}{[4,11]} \\
{[12,26]}\end{array}$ \\
\hline Fungi & Cryptococcus neoformans & Alterations in thymic architecture & [75] \\
\hline \multirow{3}{*}{ Parasites } & Trypanosoma cruzi & $\begin{array}{l}\text { Atrophy (strain dependent); release of DP/DN/autoreactive } \\
\text { T cells; alterations in extracellular matrix }\end{array}$ & {$[24,46,47,67]$} \\
\hline & Plasmodium berghei & $\begin{array}{l}\text { Atrophy (strain dependent); release of DP/DN cells; alterations } \\
\text { in extracellular matrix }\end{array}$ & {$[44,45]$} \\
\hline & Toxoplasma gondii & Atrophy & [22] \\
\hline
\end{tabular}

airus, bacteria, fungi and parasites have been documented to infect the thymus, either in humans or experimental animal models. The major consequences of each infection on thymic structure or function are listed, when information is available. 


\section{Box 1. Mechanisms of thymic atrophy}

Thymic involution is a physiological process that occurs naturally and progressively with aging and also transiently during pregnancy and stress. Atrophy occurs by a gradual and progressive decline in the number of thymocytes, and known mechanisms of physiological atrophy are mediated by either TEC or thymocytes (reviewed by Dooley et al. [20]). Because interactions between developing thymocytes and structural components of the thymus are essential for normal T cell differentiation, alterations in TEC can result in diminished functional thymopoiesis and export of naïve $T$ cells, leading to thymic atrophy. For example, thymic atrophy depends on sex hormones in a process that requires the expression of the androgen/estrogen receptors by TEC. By contrast, thymocytemediated atrophy is usually the result of alterations in the thymic milieu that induce rapid apoptotic cell death of double positive (DP) thymocytes. This is best exemplified by stress-mediated thymic involution, which occurs by DP thymocyte death in response to elevated levels of circulating GCs.

Several non-physiological stimuli can induce premature thymic atrophy, such as infection. Infection-induced atrophy could occur by: alterations in TECs; induction of apoptosis in thymocytes particularly DP cells; or reduction of thymocyte precursors within the bone marrow. The similarities between the mediators of physiological and infection-induced thymic atrophy suggest that the molecular mechanisms responsible for the decline in thymic cellularity might be common in both settings, and this possibility should be addressed experimentally (Box 2).

Why thymic atrophy should accompany infection is debated. The different hypotheses include: (i) thymic atrophy is a by-product of infection, with no specific advantage for the pathogen or the host; (ii) thymic atrophy is a virulence strategy employed by pathogens to subvert antimicrobial immunity; and (iii) thymic atrophy is a host strategy that reduces thymic activity during infection to prevent disruption of $\mathrm{T}$ cell selection and prevent the emergence of central tolerance to the invading organism. In any case, thymic atrophy can impair thymic function and has implications for ongoing immunity.

double-positive (DP) thymocytes [20]. Adrenalectomy prior to infection prevents thymocyte depletion in rabies virus infected mice, which confirms a role for GCs in infectioninduced thymic atrophy [21]. Infection-induced premature thymic atrophy also occurs independently of increased systemic GC levels. For example, adrenalectomy prior to Toxoplasma gondii infection abolishes peripheral lymphopenia but does not prevent thymocyte loss [22]. In some infections, GCs synergize with other mediators to induce thymic atrophy. These include tumor necrosis factor (TNF) during Francisella tularensis [23] and Trypanosoma cruzi [24] infection, interferon (IFN) $\gamma$ during Salmonella enterica infection [25], and IFN $\gamma$ and nitric oxide during Mycobacterium avium infection [26]. Moreover, some of these molecules can alter thymic populations independently of GCs. This is the case for TNF, which can lead to the deletion of DP thymocytes, mediated by excessive peripheral $\mathrm{T}$ cell activation following antigen injection, even after treatment with antagonists of GC receptors [27].

Interestingly, infection-induced thymic atrophy often correlates with strain virulence, as observed for T. cruzi [28], F. tularensis [23], M. avium [26], measles virus [29], highly pathogenic avian influenza viruses (HPAIVs) [7], and simian immunodeficiency virus (SIV) [30]. These data suggest that specific microbial factors directly promote thymocyte death. This is true for bacterial factors such as lipopolysaccharide [31], Escherichia coli enterotoxin [32], and mycobacterial cord factor [33], and has been confirmed with the fungal virulence factors gliotoxin [34] and toxin T-2 [35]; all of which directly induce thymocyte apoptosis when administered to mice. Local thymic effects are also observed after HIV infection [2]. HIV thymotropic viral variants are detected in vivo [36], raising the possibility that these strains would be more likely to affect thymic function. The infected cell type depends on viral tropism for chemokine CXC receptor (CXCR) 4 and chemokine CC receptor (CCR)5, but most thymocyte subsets can become infected [2]. HIV also infects different thymic stromal cells, including macrophages, conventional dendritic cells (cDCs), plasmacytoid DCs (pDCs), and thymic epithelial cells (TECs) [37-39]. HIV affects the fate of infected cell types differently. For example, CD4 single positive (SP) thymocyte depletion results from direct infection, killing of progenitor cells, and apoptosis induction of uninfected cells by viral products [40]. HIV infection also induces DC and TEC death [37,39]. In the case of DCs, cell death is associated with IFN $\alpha$ production by pDCs but not cDCs [37]. Thus, in addition to inducing thymocyte death, HIV disrupts thymic function by altering the local microenvironment. Collectively, these data show that HIV infection induces thymic atrophy and impacts thymic function, leading to a decline in the export of newly differentiated T cells $[2,41]$.

Similar to HIV, murine leukemia virus (MLV) has specific long terminal repeat region sequences that affect viral infection and replication in DP and double negative (DN) thymocytes and in other thymic populations [42]. MLV induces apoptosis of infected cells within the thymus even before the leukemic period. Apoptosis is induced by the accumulation of Env protein precursors, triggering endoplasmic reticulum stress [43]. Thus, apoptosis is a consequence of local infection, and infected DP thymocytes die more than uninfected cells within the thymus.

Thymic atrophy is also detected during infection of mice with HPAIV [7]. These viruses cause severe human disease accompanied by profound lymphopenia. After intranasal challenge of mice, influenza-infected DCs are present in the corticomedullary region of the thymus. Thymic atrophy is strain dependent and occurs only after infection with highly pathogenic virus. HPAIV interferes with thymic function, inducing loss of DP thymocytes and diminished export of naïve $\mathrm{T}$ cells to the periphery, leading to severe lymphopenia. It is unknown what role GCs play during this acute infection and the relative contribution of local versus systemic factors to thymic atrophy cannot be ascertained.

These studies demonstrate that local and systemic effects can induce thymic atrophy during infection, and are not mutually exclusive.

\section{Thymic structure is altered by infection}

Infection induces thymic structural alterations other than atrophy (Figure 2). For example, both T. cruzi and Plasmodium berghei directly infect the thymus and induce significant changes in the extracellular matrix [19,28,44-46]. $T$. cruzi increases fibronectin and laminin deposition and chemokine CXC ligand (CXCL) 12 and chemokine CC ligand (CCL)4 production within the thymus [46,47]. During $T$. cruzi infection, expression of the fibronectin and laminin receptors [very late antigen (VLA)-4, VLA-5 and VLA-6] and CXCR4 and CCR5 is augmented on thymocytes and 
intrathymic thymocyte migration of DP cells is enhanced $[46,47] . P$. berghei affects thymocyte migration by inducing CXCL12 and CXCR4 and reducing CCL25 and CCR9 production within the thymus [45]. When analyzed ex vivo, both DN and SP cells from infected thymi migrate faster than control populations towards extracellular matrix components [45]. In both cases, these changes affect peripheral $T$ cell subsets. At the peak of T. cruzi infection, a higher frequency of immature and VLA ${ }^{\text {hi }} \mathrm{DP}$ T cells are found in the periphery [46]. Similarly, increased numbers of DN and DP $T$ cells are found in the periphery of $P$. berghei-infected mice [44].

Viral infections also induce significant changes in thymic structure by infecting stromal cells. HIV can infect TECs in vivo and lead to degeneration of these cells [39]. In vivo infection of TECs has also been described using MLV [48] and CMV [49]. Mouse hepatitis virus (MHV) [50], measles virus [51], and type-B coxsackievirus [52] have been shown to infect TECs in vitro. Interestingly, in vitro infection of human TECs with measles virus results in terminal differentiation and apoptosis of these cells [51]. By contrast, type$B$ coxsackievirus infection of human TECs in vitro does not cause damage but modulates cell function, leading to increased production of interleukin (IL)-6, granulocyte-macrophage colony-stimulating factor (GM-CSF), and leukocyte migration inhibition factor (LIF) [52]. The observation that viruses infect TECs, alter their function, and in some cases induce cell death, is especially important given the crucial role these cells play in T cell differentiation [53]. Finally, the changes in thymic cellularity observed during viral infection may be caused by depletion of TECs and secondary decline in thymocyte number.

These data show that structural alterations of the thymus caused by infection modify the characteristics of differentiating $\mathrm{T}$ cells and affect $\mathrm{T}$ cell export.

\section{Alterations in thymic export}

The appearance of DP thymocytes in the periphery, as described following $T$. cruzi and P. berghei infection, is also a hallmark of infections caused by HIV, hepatitis B virus (HBV), and hepatitis C virus (HCV) $[54,55]$. MHV can be detected within the thymus [56] (Table 1), and although the origin of peripheral DP cells is unknown, one possibility is that they are released from the thymus due to local infection (Figure 2). Alternately, activated $\mathrm{T}$ cells may acquire a DP phenotype as observed during HIV and other infections [57]. Whether peripheral DP cells are an indication of altered thymic function or whether they represent activated $\mathrm{T}$ cells likely varies according to the infecting agent and requires experimental investigation.

Alterations in thymic structure due to infection can alter $\mathrm{T}$ cell export in other ways. A major consequence of HIV infection is reduced export of recent thymic emigrants; an effect confirmed by $\mathrm{T}$ cell rearrangement excision circle (TREC) analysis [2,41]. As thymic activity is essential to maintain or reconstitute a functional peripheral $\mathrm{T}$ cell pool, interventions that enhance this process may potentiate the beneficial effects of antiretroviral therapy on immunity [58].

Antigen-driven responses in the thymus can also suppress $\mathrm{T}$ cell export. Although not formally demonstrated in the context of infection, intravenous or intrathymic injection of foreign antigen leads to a significant reduction in the amount of recent thymic emigrants in the periphery [59]. This is not due to increased negative selection of developing thymocytes, but rather from retention of medullary thymocytes within the thymus [59].

Although it is intuitive that reduced thymic T cell export is a consequence of infection-induced thymic atrophy, this is not always the case. During Salmonella-induced thymic atrophy, $\mathrm{T}$ cell export is maintained [60], suggesting that atrophy and a decline in thymic function might be independent. Nevertheless, maintaining thymic export of recent thymic emigrants during infectious episodes is important, because it positively impacts ongoing immunity [14-18].

\section{Generation of T cells tolerant to pathogens}

Infection alters thymic structure and affects export of immature and naïve T cells. Another important question is whether infection of the thymus and the presence of microbial antigens in the thymus, particularly during persistent infection, leads to pathogen-specific tolerance (Figure 2). This question was investigated using a model of mycobacterial infection. Infection of mice with $M$. avium of intermediate virulence leads to chronic persistent infection. During this infection, M. avium disseminates to, and persists in, the medulla and corticomedullary region of the thymus [11,12]. No thymic atrophy occurs and the chronically infected thymus continues to support $\mathrm{T}$ cell differentiation. However, $\mathrm{T}$ cells that mature within infected thymi are abnormal because they suboptimally respond to $M$. avium antigens compared to $\mathrm{T}$ cells that differentiate in uninfected thymi. Importantly, $\mathrm{T}$ cells that differentiate in M. avium-infected thymi respond normally to unrelated antigens, indicating that the defect is specific for the invading pathogen [11]. Although the precise mechanisms responsible for this difference is still being elucidated (Box 2), these data demonstrate that thymic infection can induce pathogen-specific $\mathrm{T}$ cell tolerance.

Whether tolerance also emerges in the setting of chronic M. tuberculosis infection is a matter of debate. At various times after M. tuberculosis infection, Reiley et al. treated mice with the myeloablative drug busulfan and then infused bone marrow from ESAT6-specific TCR transgenic mice [5]. ESAT6 is a protein secreted by M. tuberculosis and is an immunodominant antigen recognized by human and murine $\mathrm{T}$ cells. This established mixed bone marrow chimeras containing a population of congenically marked ESAT6specific naïve $\mathrm{CD} 4^{+} \mathrm{T}$ cells. When performed 30 days after infection, a significant population of congenic ESAT6-specific $\mathrm{CD} 4^{+} \mathrm{T}$ cells appeared in lungs within 49 days after reconstitution and gradually increased during the next 3 months. However, if the bone marrow infusion was delayed until 3 months after infection, few if any ESAT6-specific $\mathrm{CD}^{+} \mathrm{T}$ cells could be detected in the lung 49 and 63 days after reconstitution, and only began to appear 84 days after infection, reaching steady-state levels by 140 days. This result was interpreted as indicating that recent thymic emigrants help maintain the peripheral $\mathrm{T}$ cell response to $M$. tuberculosis; central tolerance to $M$. tuberculosis antigens does not occur. The authors suggested that the decline in the expansion of congenic recent thymic emigrants after day 90 was due to diminished priming and/or expansion of 
naïve $\mathrm{T}$ cells during chronic infection. However, an alternative interpretation of their data is that $M$. tuberculosis colonization of the thymus leads to tolerance. Few bacteria are present in the thymus during the first month after infection; by contrast, nearly all infected mice have a significant bacterial load by 3 months $[4,5,12]$. The diminished expansion of primed ESAT6-specific $\mathrm{CD} 4^{+} \mathrm{T}$ cells late after infection (d90) can be explained by the progressive development of tolerance during chronic mycobacterial infection, which occurs only when there is a significant amount of mycobacterial antigen in the thymus. Clearly, whether infection-induced central tolerance contributes to the inability of recent thymic emigrants to integrate the immune response during chronic infection with M. tuberculosis requires further investigation.

Tolerance to invading pathogens is also observed during viral infection. Neonatal MLV infection leads to infection of thymocytes as well as thymic stromal cells, and renders T cells tolerant to viral antigens [61]. Similarly, congenitally acquired lymphocytic choriomeningitis virus (LCMV) infection is a model of immune tolerance: mice infected in utero or at birth show high viral titers in most organs, including the thymus, and have a selective defect in LCMV-specific T cell immunity [62]. LCMV infection of the thymus starts at the fetal stage in DN cells, and transitions to CD4 SP cells in the adult thymus. By contrast, $\mathrm{CD}^{+} \mathrm{T}$ cell infection is minimal [63], and transferred virus-specific cytotoxic T lymphocytes (CTLs), from immunized mice, infiltrate the thymus and eliminate the infection $[6,62]$. In this model, viral clearance is associated with reacquisition of LCMV-specific CTL responses, suggesting that continuous presence of the antigen in the thymus is required to maintain tolerance, not only during fetal development but in adult animals as well.

Viral hepatitis also induces central tolerance to HBV. Although acute infection in adults is readily resolved, HBV infection in utero induces tolerance to viral proteins [64], and infants born to HBV-infected mothers are more likely to become chronic carriers of HBV [65]. One possibility is that viral proteins in the neonatal thymus induce HBV-specific $\mathrm{T}$ cell tolerance. This hypothesis is supported by the observation that both MLV and HBV infect TECs [50,61], which could explain why these infections induce $\mathrm{T}$ cell tolerance while others do not. TEC turnover is rapid [66], therefore, this hypothesis implies that these viruses can continually infect new TECs or reside in thymic epithelial stem cells.

These data suggest that direct infection of the thymus by viruses and bacteria alter $\mathrm{T}$ cell selection and induce tolerance against the invading pathogen, with the potential to impair ongoing immunity. An interesting question is whether the mechanism of tolerance induced by thymic infection involves the generation of pathogen-specific regulatory $\mathrm{T}$ cells, $\mathrm{T}$ cell anergy, or negative selection of differentiating pathogen-reactive $\mathrm{T}$ cells (Box 2).

\section{Autoimmunity induced by thymic infection}

Pathogens that disrupt thymic function may also disrupt the development of central tolerance. Autoreactive T cells that are normally negatively selected in the thymus might escape death because of thymic dysfunction, emigrate to the periphery, and trigger autoimmunity. Although this

\section{Box 2. Outstanding questions}

- How universal is thymic infection? Do other pathogens infect the thymus?

- How does the thymus get infected? Do pathogens directly target the thymus, or do they disseminate inside recirculating cells?

- Why does atrophy accompany thymic infection so frequently? Is atrophy beneficial for the host or the bug? What are the mechanisms responsible for infection-induced thymic atrophy?

- What are the mechanisms responsible for T cell tolerance? Does the presence of microbial antigens lead to negative selection of developing T cells? Do microbe-specific regulatory $T$ cells emerge following thymic infection? Are developing $T$ cells anergic to the infectious agent?

- How does infection of the thymus impact ongoing immunity? Does thymic infection during childhood impact immunity later in life? Is thymic infection relevant during vaccination with live microorganisms?

- How important are newly generated T cells during chronic infections and during immune reconstitution?

possibility remains theoretical, some data support it. During experimental $T$. cruzi infection, T cells expressing 'forbidden' TCRs that are normally deleted in the thymus, particularly those belonging to the V $\beta 5$ and V $\beta 12$ families, survive and can be detected in peripheral lymph nodes [67]. Thymic SP cells from infected mice are not enriched in those TCRs. These data indicate that the appearance of these forbidden TCRs in the periphery is not due to defective negative selection but a consequence of abnormal migration of immature cells [67]. In addition, anti-thymus antibodies and myocardium-specific autoreactive $\mathrm{T}$ cells are detected following $T$. cruzi infection [28], raising the possibility that infection-induced thymic alterations potentiate autoimmunity.

\section{Thymic infection: the beginning and the end}

How are various microbes able to reach the thymus and establish infection? Furthermore, if dissemination to the thymus occurs commonly during infection, has the immune system evolved mechanisms to respond to pathogens invading the thymus?

\section{How do microorganisms reach the thymus?}

Two scenarios are possible when considering the origin of thymic infection during hematogenous spread of infection (Figure 3; Box 2). First, circulating pathogens can enter the thymus and infect cells in a targeted manner, as represented by thymotropic variants of HIV [36] and MLV [42]. Alternately, there is the 'Trojan Horse' model. The trafficking of several cell types between the periphery and the thymus make this possible. $\mathrm{T}$ cells circulate from the periphery to the thymus [68], and if infected, could seed the thymus with pathogens that target T cells (e.g., HIV). Similarly, certain DC subsets (e.g., Sirp $\alpha^{+} \mathrm{cDCs}$ ) migrate from the periphery to the thymus and modulate $\mathrm{T}$ cell tolerance [69]. DCs infected with influenza virus or $M$. avium can be detected within the thymus, raising the possibility that infected DCs spread the infection [7,11]. DCs are responsible for disseminating $M$. tuberculosis from the lung to the draining lymph nodes [70], and it is possible that dissemination to the thymus occurs by a similar mechanism. Together, these data support the idea that cells circulating to the thymus can carry infectious agents 


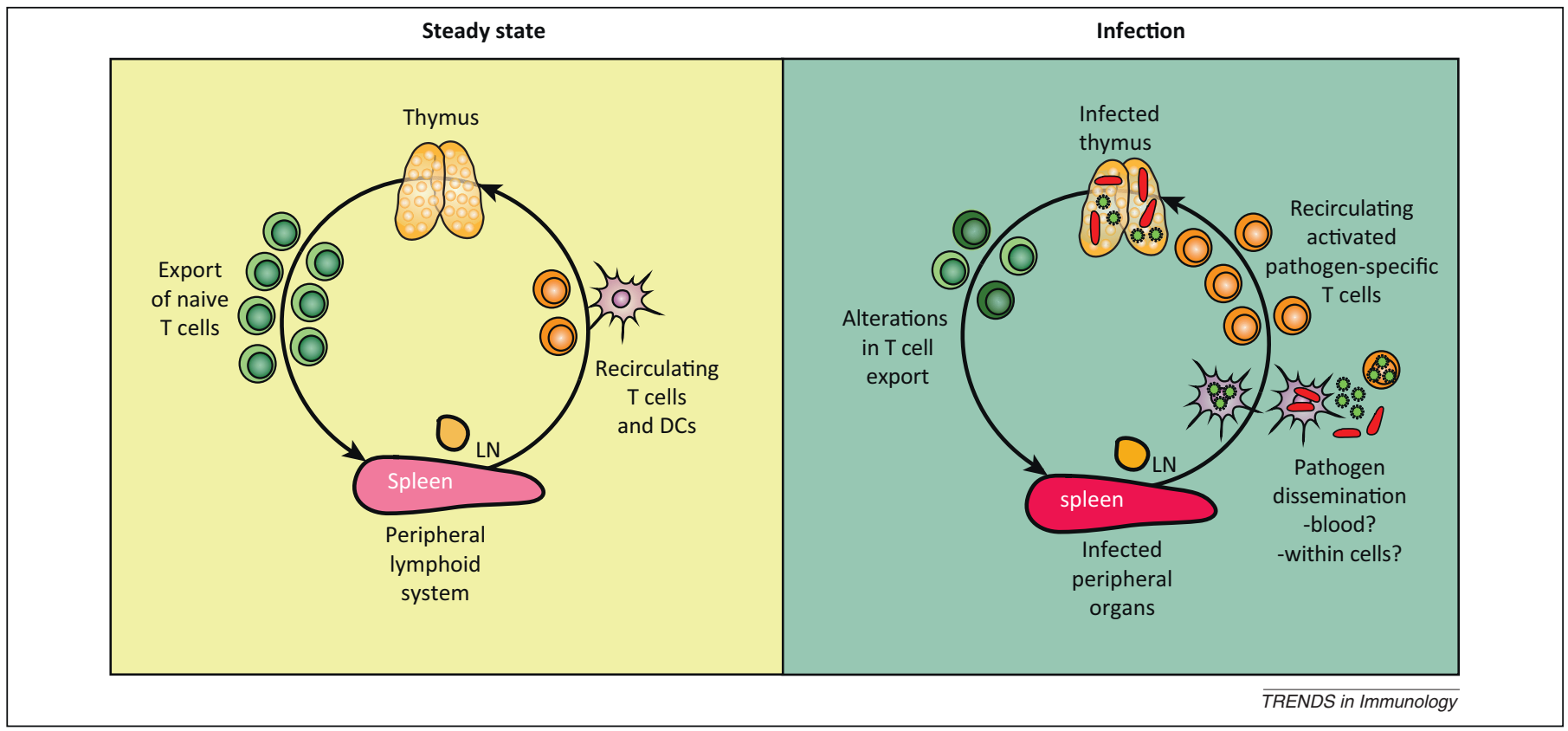

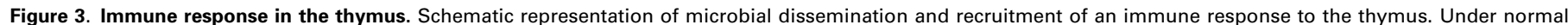
conditions, mature T cells and DC circulate from peripheral lymphoid organs to the thymus (left pane/). Following infection, pathogens disseminate from the periphery to

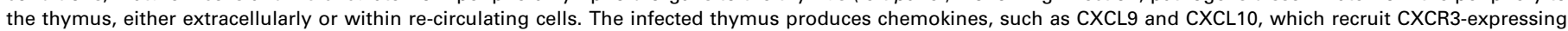
antigen-specific $T$ cells from the peripheral tissues back to the thymus to fight infection (right panel). Abbreviation: $L N$, lymph node.

and seed thymic infection (Figure 3). An important corollary is that the route and duration of infection may modify the risk that the thymus becomes infected by different pathogens.

\section{Immunity within the thymus}

If the thymus is a site of infection, it may recruit an immune response against invading pathogens. As discussed, during systemic LCMV infection, LCMV-specific CTLs traffic to the thymus, establish an immune response, and eliminate LCMV from the thymus [6,62]. Similarly, during influenza infection, functional influenza-specific CTLs are detected within the thymus [7]. Finally, antigen-specific $\mathrm{CD}^{+}$and $\mathrm{CD}^{+} \mathrm{T}$ cells are detected in the thymus following $M$. avium and $M$. tuberculosis infection, as part of the immune response against persistent bacteria $[4,5]$. The responding $\mathrm{T}$ cells in the thymus are not newly differentiated mature thymocytes but instead are activated $\mathrm{T}$ cells that circulate from peripheral organs to the thymus to fight infection (Figure 3) [4]. Under these conditions, the recruitment of activated T cells is associated with increased expression of T helper 1 chemokines and an enrichment of $\mathrm{CXCR}^{+}$mycobacteria-specific $\mathrm{T}$ cells within the thymus [4]. These results confirm that the thymus is not only a site of infection, but suggest that it is actively surveyed by the immune system.

\section{Concluding remarks}

The recent studies reviewed here show that the thymus is a site of infection that has important immunological consequences. Pathogens disrupt thymic structure and function, and alter $\mathrm{T}$ cell selection and export. These changes affect the peripheral $\mathrm{T}$ cell pool and affect ongoing and future immune responses.

These data suggest a model in which the effect of infection on the thymus depends on the type of microbe, the severity of infection, and the ability of the pathogen to infect and persist within the infected thymus (Figure 2). In this scheme, acute infection is characterized by increased GCs and proinflammatory mediator levels, which can lead to thymic atrophy. This effect is more pronounced in DP cells and can occur even in the absence of the pathogen in the thymus. Local thymic infection can exacerbate atrophy, through remodeling of extracellular matrix, production of virulence factors, or direct infection of thymic cells. These structural changes affect thymic function; particularly $\mathrm{T}$ cell export, leading to the release of immature (DP/DN) or autoreactive T cells into the periphery. Despite the profound effects of acute infection on the thymus, the impact of thymic dysfunction on immunity is predicted to be limited and transient because the peripheral $\mathrm{T}$ cell pool should include pre-existing pathogen-specific T cells. By contrast, local thymic infection may have severe repercussions, particularly for: (i) infections acquired during childhood - when the $\mathrm{T}$ cell repertoire is still developing; (ii) in the setting of persistent infection (e.g., tuberculosis), which may induce T cell tolerance; and (iii) infections associated with severe lymphopenia (e.g., HIV), when lymphoid reconstitution is required. In these cases, emergence of central tolerance to the infectious agent may impair deployment of pathogen-reactive $\mathrm{T}$ cells in the naïve repertoire. Such a scenario could favor the microbe, because impairment of T cell immunity would contribute to pathogen persistence. In order to minimize such consequences, mechanisms exist to respond to direct infection of the thymus (Figure 3). Just as in other tissues, these rely on the trafficking of peripheral $\mathrm{T}$ cells from secondary lymphoid tissue back to the thymus. Although required for protection, circulation of cells back to the thymus could allow some pathogens access to the thymus.

Altogether, preserving a sterile thymic environment is important to maintain thymic integrity, both structurally 
and functionally, sustain optimal $\mathrm{T}$ cell differentiation and export, and prevent the emergence of tolerance to invading pathogens. Therefore, the thymus should be regarded as an active player during infectious episodes and the contribution of this organ for ongoing immunity should be addressed in future studies (Box 2).

\section{Acknowledgments}

We thank Joana Neves and Nadine Santos for critical reading of the manuscript. This work was supported by Portuguese Foundation for Science and Technology (FCT) grant PTDC/SAU-MII/101663/2008 and individual fellowships to CN-A and CN. SMB was supported by National Institutes of Health Grant R01 R56 AI067731.

\section{References}

1 Starr, T.K. et al. (2003) Positive and negative selection of T cells. Annu. Rev. Immunol. 21, 139-176

2 Ho Tsong Fang, R. et al. (2008) The role of the thymus in HIV infection: a 10 year perspective. AIDS 22, 171-184

3 Raviola, E. and Karnovsky, M.J. (1972) Evidence for a blood-thymus barrier using electron-opaque tracers. J. Exp. Med. 136, 466-498

4 Nobrega, C. et al. (2013) T cells home to the thymus and control infection. J. Immunol. 190, 1646-1658

5 Reiley, W.W. et al. (2012) Maintenance of peripheral T cell responses during Mycobacterium tuberculosis infection. J. Immunol. 189, 44514458

6 Gossmann, J. et al. (1991) Entry of antivirally active T lymphocytes into the thymus of virus-infected mice. J. Immunol. 146, 293-297

7 Vogel, A.B. et al. (2010) Highly pathogenic influenza virus infection of the thymus interferes with T lymphocyte development. J. Immunol. $185,4824-4834$

8 Freitas, A.A. and Rocha, B.B. (1993) Lymphocyte lifespans: homeostasis, selection and competition. Immunol. Today 14, 25-29

9 World Health Organization (2013) Global health observatory. http:// www.who.int/gho/child_health/en/

10 Prabhu, A.D. et al. (2008) Tuberculosis of thymus - a case report. Heart Lung Circ. 17, 345-346

11 Nobrega, C. et al. (2010) Dissemination of mycobacteria to the thymus renders newly generated T cells tolerant to the invading pathogen. $J$. Immunol. 184, 351-358

12 Nobrega, C. et al. (2007) The thymus as a target for mycobacterial infections. Microbes Infect. 9, 1521-1529

13 Sauce, D. et al. (2009) Evidence of premature immune aging in patients thymectomized during early childhood. J. Clin. Invest. 119, 3070-3078

14 Vezys, V. et al. (2006) Continuous recruitment of naive T cells contributes to heterogeneity of antiviral CD8 T cells during persistent infection. J. Exp. Med. 203, 2263-2269

15 Miller, N.E. et al. (2005) Role of thymic output in regulating CD8 T-cell homeostasis during acute and chronic viral infection. J. Virol. 79, 9419-9429

16 Pellegrini, M. et al. (2011) IL-7 engages multiple mechanisms to overcome chronic viral infection and limit organ pathology. Cell 144, 601-613

17 AbuAttieh, M. et al. (2012) Affinity maturation of antibodies requires integrity of the adult thymus. Eur. J. Immunol. 42, 500-510

18 Song, F. et al. (2006) The thymus plays a role in oral tolerance in experimental autoimmune encephalomyelitis. J. Immunol. 177, 15001509

19 Savino, W. (2006) The thymus is a common target organ in infectious diseases. PLoS Pathog. 2, e62

20 Dooley, J. and Liston, A. (2012) Molecular control over thymic involution: from cytokines and microRNA to aging and adipose tissue. Eur. J. Immunol. 42, 1073-1079

21 Cardenas Palomo, L.F. et al. (1995) Lymphocyte subsets and cell proliferation analysis in rabies-infected mice. J. Clin. Lab. Immunol. $46,49-61$

22 Huldt, G. et al. (1973) Effect of Toxoplasma gondii on the thymus. Nature 244, 301-303

23 Chen, W. et al. (2005) Low dose aerosol infection of mice with virulent type A Francisella tularensis induces severe thymus atrophy and CD4+CD8+ thymocyte depletion. Microb. Pathog. 39, 189-196
24 Perez, A.R. et al. (2007) Thymus atrophy during Trypanosoma cruzi infection is caused by an immuno-endocrine imbalance. Brain Behav. Immun. 21, 890-900

25 Deobagkar-Lele, M. et al. (2012) Interferon gamma and glucocorticoid mediated pathways synergize to enhance death of CD4(+) CD8(+) thymocytes during Salmonella enterica serovar Typhimurium infection. Immunology 138, 307-321

26 Borges, M. et al. (2012) Molecular and cellular mechanisms of Mycobacterium avium-induced thymic atrophy. J. Immunol. 189, $3600-3608$

27 Martin, S. and Bevan, M.J. (1997) Antigen-specific and nonspecific deletion of immature cortical thymocytes caused by antigen injection. Eur. J. Immunol. 27, 2726-2736

28 Savino, W. et al. (1989) Studies on the thymus in Chagas' disease. I. Changes in the thymic microenvironment in mice acutely infected with Trypanosoma cruzi. Eur. J. Immunol. 19, 1727-1733

29 Auwaerter, P.G. et al. (1996) Measles virus infection of thymic epithelium in the SCID-hu mouse leads to thymocyte apoptosis. $J$. Virol. 70, 3734-3740

30 Wykrzykowska, J.J. et al. (1998) Early regeneration of thymic progenitors in rhesus macaques infected with simian immunodeficiency virus. J. Exp. Med. 187, 1767-1778

31 Baroni, C.D. et al. (1976) Biological effects of Escherichia coli lipopolysaccharide (LPS) in vivo. I. Selection in the mouse thymus of killer and helper cells. Immunology 31, 217-224

32 Tsuji, T. et al. (2000) Induction of apoptosis in lymphoid tissues of mice after intramuscular injection of enterotoxigenic Escherichia coli enterotoxin. Immunobiology 201, 377-390

33 Ozeki, Y. et al. (1997) In vivo induction of apoptosis in the thymus by administration of mycobacterial cord factor (trehalose 6,6'-dimycolate). Infect. Immun. 65, 1793-1799

34 Sutton, P. et al. (1994) In vivo immunosuppressive activity of gliotoxin, a metabolite produced by human pathogenic fungi. Infect. Immun. 62 , 1192-1198

35 Islam, Z. et al. (1998) T-2 toxin induces thymic apoptosis in vivo in mice. Toxicol. Appl. Pharmacol. 148, 205-214

36 Calabro, M.L. et al. (1995) HIV-1 infection of the thymus: evidence for a cytopathic and thymotropic viral variant in vivo. AIDS Res. Hum. Retroviruses 11, 11-19

37 Schmitt, N. et al. (2006) Differential susceptibility of human thymic dendritic cell subsets to X4 and R5 HIV-1 infection. AIDS 20, 533-542

38 Rozmyslowicz, T. et al. (2010) HIV-1 infection inhibits cytokine production in human thymic macrophages. Exp. Hematol. 38, 1157-1166

39 Stanley, S.K. et al. (1993) Human immunodeficiency virus infection of the human thymus and disruption of the thymic microenvironment in the SCID-hu mouse. J. Exp. Med. 178, 1151-1163

$40 \mathrm{Su}$, L. et al. (1995) HIV-1-induced thymocyte depletion is associated with indirect cytopathogenicity and infection of progenitor cells in vivo. Immunity 2, 25-36

41 Douek, D.C. et al. (2001) Evidence for increased T cell turnover and decreased thymic output in HIV infection. J. Immunol. 167, 6663-6668

42 Yoshimura, F.K. et al. (1999) Sequences between the enhancer and promoter in the long terminal repeat affect murine leukemia virus pathogenicity and replication in the thymus. J. Virol. 73, 4890-4898

43 Yoshimura, F.K. and Luo, X. (2007) Induction of endoplasmic reticulum stress in thymic lymphocytes by the envelope precursor polyprotein of a murine leukemia virus during the preleukemic period. J. Virol. 81, 4374-4377

44 Francelin, C. et al. (2011) Effects of Plasmodium berghei on thymus: high levels of apoptosis and premature egress of CD4(+)CD8(+) thymocytes in experimentally infected mice. Immunobiology $216,1148-1154$

45 Gameiro, J. et al. (2010) Changes in cell migration-related molecules expressed by thymic microenvironment during experimental Plasmodium berghei infection: consequences on thymocyte development. Immunology 129, 248-256

46 Cotta-de-Almeida, V. et al. (2003) Trypanosoma cruzi infection modulates intrathymic contents of extracellular matrix ligands and receptors and alters thymocyte migration. Eur. J. Immunol. 33, 24392448

47 Mendes-da-Cruz, D.A. et al. (2006) Altered thymocyte migration during experimental acute Trypanosoma cruzi infection: combined role of fibronectin and the chemokines CXCL12 and CCL4. Eur. J. Immunol. 36, 1486-1493 
48 Feldman, D.G. et al. (1967) Electron microscopic study of the mouse leukemia virus (Gross) in organs of mouse embryos from virus-injected and normal C3Hf parents. Cancer Res. 27, 1792-1804

49 Mocarski, E.S. et al. (1993) Human cytomegalovirus in a SCID-hu mouse: thymic epithelial cells are prominent targets of viral replication. Proc. Natl. Acad. Sci. U.S.A. 90, 104-108

50 Lamontagne, L. and Jolicoeur, P. (1994) Low-virulent mouse hepatitis viruses exhibiting various tropisms in macrophages, $\mathrm{T}$ and $\mathrm{B}$ cell subpopulations, and thymic stromal cells. Lab. Anim. Sci. 44, 17-24

51 Valentin, H. et al. (1999) Measles virus infection induces terminal differentiation of human thymic epithelial cells. J. Virol. 73, 2212-2221

52 Brilot, F. et al. (2002) Persistent infection of human thymic epithelial cells by coxsackievirus B4. J. Virol. 76, 5260-5265

53 Takahama, Y. (2006) Journey through the thymus: stromal guides for T-cell development and selection. Nat. Rev. Immunol. 6, 127-135

54 Weiss, L. et al. (1998) Persistent expansion, in a human immunodeficiency virus-infected person, of $\mathrm{V}$ beta-restricted CD4+CD8+ $\mathrm{T}$ lymphocytes that express cytotoxicity-associated molecules and are committed to produce interferon-gamma and tumor necrosis factor-alpha. J. Infect. Dis. 178, 1158-1162

55 Nascimbeni, M. et al. (2011) Distinct CD4+ CD8+ double-positive T cells in the blood and liver of patients during chronic hepatitis $\mathrm{B}$ and $\mathrm{C}$. PLoS ONE 6, e20145

56 Godfraind, C. et al. (1995) Thymus involution induced by mouse hepatitis virus A59 in BALB/c mice. J. Virol. 69, 6541-6547

57 Nascimbeni, M. et al. (2004) Peripheral CD4(+)CD8(+) T cells are differentiated effector memory cells with antiviral functions. Blood 104, 478-486

58 Corbeau, P. and Reynes, J. (2011) Immune reconstitution under antiretroviral therapy: the new challenge in HIV-1 infection. Blood $117,5582-5590$

59 Uldrich, A.P. et al. (2006) Antigen challenge inhibits thymic emigration. J. Immunol. 176, 4553-4561

60 Ross, E.A. et al. (2012) Thymic function is maintained during Salmonella-induced atrophy and recovery. J. Immunol. 189, 4266-4274

61 Korostoff, J.M. et al. (1990) Neonatal exposure to thymotropic gross murine leukemia virus induces virus-specific immunologic nonresponsiveness. J. Exp. Med. 172, 1765-1775
62 Jamieson, B.D. and Ahmed, R. (1988) T-cell tolerance: exposure to virus in utero does not cause a permanent deletion of specific $\mathrm{T}$ cells. Proc. Natl. Acad. Sci. U.S.A. 85, 2265-2268

63 Ahmed, R. et al. (1987) Virus-lymphocyte interaction: T cells of the helper subset are infected with lymphocytic choriomeningitis virus during persistent infection in vivo. J. Virol. 61, 1571-1576

64 Milich, D.R. et al. (1990) Is a function of the secreted hepatitis B e antigen to induce immunologic tolerance in utero? Proc. Natl. Acad. Sci. U.S.A. 87, 6599-6603

65 Stevens, C.E. et al. (1975) Vertical transmission of hepatitis B antigen in Taiwan. N. Engl. J. Med. 292, 771-774

66 Gray, D.H. et al. (2006) Developmental kinetics, turnover, and stimulatory capacity of thymic epithelial cells. Blood 108, 3777-3785

67 Mendes-da-Cruz, D.A. et al. (2003) Experimental Trypanosoma cruzi infection alters the shaping of the central and peripheral T-cell repertoire. Microbes Infect. 5, 825-832

68 Hale, J.S. and Fink, P.J. (2009) Back to the thymus: peripheral T cells come home. Immunol. Cell Biol. 87, 58-64

69 Proietto, A.I. et al. (2009) The impact of circulating dendritic cells on the development and differentiation of thymocytes. Immunol. Cell Biol. 87, 39-45

70 Wolf, A.J. et al. (2007) Mycobacterium tuberculosis infects dendritic cells with high frequency and impairs their function in vivo. $J$. Immunol. 179, 2509-2519

71 Cavalcante, P. et al. (2010) Epstein-Barr virus persistence and reactivation in myasthenia gravis thymus. Ann. Neurol. 67, 726-738

72 Calello, M.A. et al. (1986) Relationship between Junin virus infection of thymus and the establishment of persistence in rodents. Med. Microbiol. Immunol. 175, 109-112

73 Cavalcante, P. et al. (2010) Detection of poliovirus-infected macrophages in thymus of patients with myasthenia gravis. Neurology 74, 1118-1126

74 Brito, V.N. et al. (2003) Thymus invasion and atrophy induced by Paracoccidioides brasiliensis in BALB/c mice. Med. Mycol. 41, 83-87

75 Sotomayor, C.E. et al. (1995) Immunosuppression in experimental cryptococcosis: variation of splenic and thymic populations and expression of class II major histocompatibility complex gene products. Clin. Immunol. Immunopathol. 77, 19-26 